\title{
Discussing Chronic Poverty Issues in Ethnic Rural Areas
}

\author{
Lingling Qiu, a, Fan Yang 2,b, Weizhong Zeng ${ }^{3, c, *}$
}

${ }^{1}$ College of Economics, Sichuan Agricultural University, No.211 Huimin Road, Wenjiang District, Chengdu, 611130 China

${ }^{2}$ College of Management, Sichuan Agricultural University, No.211 Huimin Road, Wenjiang

District, Chengdu, 611130 China

${ }^{3}$ College of Economics, Sichuan Agricultural University, No.211 Huimin Road, Wenjiang District, Chengdu, 611130 China

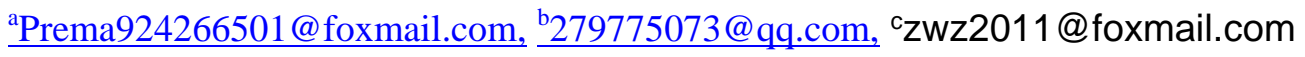

Keywords: Ethnic rural areas, Chronic poverty, Long-term, inter-generational transmission

Abstract. ethnic rural areas consist of old, young, remote and poverty-stricken regions, which are critical in poverty alleviation. Existing studies have shown that chronic poverty is more prevailing in ethnic regions, and it should be highly emphasized. On the basis of introducing the chronic poverty theories, this article took two typical areas as an example, illustrating the primary features of chronic poverty in ethnic rural areas, and subsequently put forward some relevant countermeasures and suggestions to solve the problems caused by chronic poverty.

\section{Introduction}

It is the essential requirement of socialism to eliminate poverty, improve people's livelihood and achieve common prosperity. To achieve the goal of "building a well-off society in an all-round way in 2020", it is most crucial to vigorously promote the policy of targeted poverty alleviation in povertystricken areas, especially in ethnic rural areas. Only when poverty is eliminated in poverty-stricken areas can the goal of building a well-off society in an all-round way be accomplished. Fully building a well-off society should focus on rural areas, and struggle hard in the ethnic rural areas. Constrained by the regional ecological environment conditions and resource endowments, socio-economic development of ethnic rural areas is lagging behind, chronic poverty of which is serious, becoming the problem which is most difficult to overcome at the present stage of poverty alleviation. In-depth study of the problem of chronic poverty in ethnic rural areas plays an essential role in implementing strategic measures of targeted poverty alleviation and maintaining the prosperity and stability.

Since the end of the twentieth century, chronic poverty has gradually become an important area of poverty research. Chronic poverty is the state of poverty where poverty lasted for many years (usually five years or more, even throughout the poor) and may pass on poverty to the next generation [1]. It was in "China's rural poverty monitoring report (2002)" that China's poverty situation had been divided into temporary poverty and chronic poverty for the first time. Jalan and Ravallion categorized poverty into chronic poverty, recurrent poverty and non-poverty on the basis of the six-year panel data of China's rural household expenditure, and argued that there was chronic poverty in China, but not very typical [2]. Zhang et al. decomposed poverty into temporary poverty and chronic poverty according to the revised Rodgers method which measured poverty of families or individuals over a period of time, and clearly defined and differentiated the two poverty states, on which basis sample poor groups were classified specifically [3]. Chen measured the degree of vulnerability selecting China's ecological fragile areas as the research sample, and on this basis explored connection between the ecological environment vulnerability and the occurrence of chronic poverty [4]. On the basis of the analytic framework of poverty inter-generational transmission, Lan analyzed the poverty situation of poor households in Liangshan Yi ethnic rural areas by describing the statistics, and empirically illustrated the impacts of chronic poverty [5].

Although research on chronic poverty has been carried out to a certain depth and breadth, specific research on chronic poverty of ethnic rural areas is still relatively rare, denoting follow-up studies are 
in need. Based on the long-term poverty, this paper discusses the typical characteristics of chronic poverty in ethnic rural areas by taking Meigu County of Liangshan Yi Perfecture and Kangding County of Ganzi Tibetan Prefecture in Sichuan Province as example, and ultimately puts forward some countermeasures to solve the problem of chronic poverty in ethnic rural areas.

\section{Chronic Poverty Should be Emphasized in the Process of Poverty Alleviation in Ethnic Rural Areas}

2.1 New Challenges for Poverty Alleviation Arose in the New Era Since the reform of 'being open to the world' was carried out, China have made remarkable progress in poverty alleviation and formed a special path for poverty alleviation in ethnic rural areas. However, in the new era, with the adjustment of poverty policies by national government as well as changes of poverty situation, poverty alleviation tasks in ethnic rural areas faced new challenges. First of all, poverty alleviation is hard to proceed in ethnic regions due to the large area and dense population. In 2010, the State Council Poverty Alleviation Bureau announced that 341 out of 592 poverty-stricken counties were located in the ethnic rural areas, accounting for $57.60 \%$ of the total number of poverty-stricken counties. What's more, with the poverty line standard up, the number of poor people in ethnic rural areas increased, and the scope of poverty significantly expanded. From 2006 to 2010, rural poor population in the national autonomous areas accounted for $44.5 \%, 52.2 \%, 52.5 \%$ and $55.1 \%$ of the total rural poor population respectively, with the proportion of the poor in ethnic rural areas increasing year after year [6]. Moreover, after the problem of food and clothing was solved, ethnic rural areas encountered multiple tasks of speeding up poverty alleviation, narrowing the gap between the rich and the poor, improving the regional ecological environment, maintaining regional stability and improving the development capacity. Most of the ethnic rural areas were situated in those regions whose development was restricted or prohibited by national government, with fragile ecological environment and great difficulty in exploiting. In recent years, the progress of poverty reduction in ethnic rural areas has gradually slowed down, and the poverty incidence rate of rural poor people has been increasing with the problem of decreasing poverty rate more severe.

2.2 Chronic Poverty in Ethnic Rural Areas Is Common and Urgent to Be Coped With Since the reform of opening up was implemented, although remarkable achievement has been achieved for socio-economic development and rural poverty alleviation in the ethnic rural areas, due to constraints of geographical conditions, resource endowments, ecological environment and other conditions, the development of ethnic rural areas was still lagging behind, with large amounts of rural people trapped into long-term poverty. In ethnic rural areas there are a lot of poor people whose ancestors were poor, following production techniques and lifestyles of predecessors. It is an indisputable fact that poverty in ethnic rural areas is relatively chronic compared to non-ethnic rural areas.

\section{Main Achievements and Shortcomings of Research on Chronic Poverty}

3.1 Having Identified the Multidimentional Nature of Poverty The study of chronic poverty inherited the thinking and methods of studying poverty from a multidimensional perspective. The chronic poor population not only have low levels of economic income, but also have many causes of poverty, such as low educational level, shortage of resources, poor natural endowment, poor geographical infrastructure and unavailability of opportunities. The deprivation of rights is one of the major reasons why poor families were suffering from chronic poverty for a long time. Over a long period, the vast impoverished areas, especially ethnic rural areas, owing to their own conditions and lack of right awareness, had no idea about how to make use of their rights empowered by the government. Information asymmetry and phenomenon that resources were captured by rural elites further weakened the rights of the poor themselves and the use of opportunities. What's mentioned above is likely to lead to consequences that the poor can not properly use their own rights and opportunities, and can not effectively pursue their own interests, having limited access to the rights given by the government, thus being trapped in helplessness and poverty in a long period. 
3.2 Having Depicted a Human Poverty Map from the Dynamic Perspective Researchers of chronic poverty have divided and discussed poverty issues from a dynamic point of view, such as analyzing poverty distribution, poverty and environment, poverty and parental inter-generational transmission, poverty and disability, poverty and vulnerability, and social exclusion. The research described above enriched the traditional poverty research which was not dynamic, and started a new chapter for the follow-up study. In the past 20 years, poor people below the poverty line in the long run worldwide has always been the research object for researchers. Through dynamically monitoring the poor population data of different regions, researchers have showed the poverty situation of different countries and different regions to numerous readers from the perspective of time series, and dynamically explored the transmission track of poverty states of poor population by comparing different time points, which ultimately depicted a clear map of poverty for follow-up researchers.

3.3 There Are Still Some Constraints for the Scope and Theories of Chronic Poverty Research As an important part of poverty research, chronic poverty research began in the late 20th century. In contrast to traditional poverty studies, chronic poverty research started relatively late. Since the beginning, although chronic poverty research has been greatly developed, there are still some drawbacks. The current research scope of chronic poverty is relatively limited, and research on the endogenous mechanism and development regularity of chronic poverty is still lacking. Many studies suggest that China is not a chronically poor country, but there is chronic poverty. So what is the cause of chronic poverty in China? What is the geographical distribution of chronic poverty in China? How to solve the problem of chronic poverty in China? These puzzles need to be further solved.

\section{Main Characteristics of Chronic Poverty in Ethnic Rural Areas--Two Cases}

Based on the field survey on Meigu County of Liangshan Yi Perfecture and Kangding County of Ganzi Tibetan Prefecture in Sichuan Province from July to August in 2016, this paper analyzed the main features of chronic poverty in both counties.

4.1 Sample Profile Meigu County is located in the southwest of Sichuan Province, northeast corner of Liangshan Yi Autonomous Prefecture, adjacent to the Hengduan Mountains in the southeastern part of the Qinghai-Tibet Plateau and the southwest edge of the Sichuan Basin. The County is mountainous, the highest altitude of which is 4042 meters, and climate there is diverse. Papu Township, the County economic center, is $170 \mathrm{~km}$ away from Xichang City, which is the capital of Liangshan Yi Autonomous Prefecture, and $385 \mathrm{~km}$ from the provincial capital, Chengdu. The county had a total population of 255,100 at the end of 2012, 249,700 of which belonged to ethnic minority, accounting for $97.89 \%$ of the total population, which is a typical ethnic county in China. The county had a poor population of 67937 , and the incidence of poverty was $26.63 \%$, higher than the average levels of both Sichuan Province and Liangshan Yi Autonomous Prefecture.

Kangding County is located in the eastern part of Ganzi Prefecture, lying in the western edge of the Sichuan Basin and the Qinghai-Tibet Plateau transition zone. The County has three townships, which are Rongcheng, Guzan and Xinduqiao respectively, and County government is seated in Rongcheng. The distance from Kangding County to Chengdu is over 370 kilometers, with 318 national highway running through the whole territory. By the end of 2013, 1.3797 million out of 6.9568 million rural residents hadn't yet gotten rid of poverty, and the incidence of poverty was $18.70 \%$.

The above two ethnic counties have common characteristics, which are that the incidence of poverty was much higher than the regional and national average level and the poor people were trapped in poverty for a long period.

4.2 Survey methods Field investigation method was used to carry out the research. First of all, we collected some information about general poverty situation from the statistics departments of the sample counties, mainly including scale of poverty-stricken population, poverty causes, geographical distribution of poverty, poverty categories, poverty duration, poverty alleviation plans, poverty reduction measures, poverty reduction effectiveness, etc. Secondly, referring to the overall economic situations of the counties, we randomly selected three villages at different development levels, which are categorized as the better, the medium and the poor village respectively, and subsequently 
implemented the on-site survey. Finally, combined with the field survey data and the macro statistics of the counties, we made analysis.

\subsection{Basic Conclusions}

4.3.1 Poverty Duration Was Relatively Long and Regional Differences Are Obvious In the two ethnic counties investigated, the state of poverty was found to have varying degrees of persistence and long term. At the end of 2012, Yiguojue Township of Meiju County, had a total population of 9019, with 2791 people's per capita annual income lower than 2376 yuan, the national poverty line in 2013 , identified as poor by the government, and the incidence of poverty was $30.95 \%$. What's more, there were 2372 people trapped into poverty for a long time, lasting for 5 years or longer, accounting for $85 \%$ of the total population of Yiguojue Township. Probably due to the fact that Yiguojue Township was greatly distant from the economic and cultural center of Meigu County, the proportion of poor people in Yiguojue Township was much higher than 23.52\%, the average level of the County. In the village called Yideamo, far from Yiguojue Township and located in the mountain, almost all of the villagers were poverty-stricken, and nearly $100 \%$ of the poor were kept in poverty for more than 5 years. At the end of 2013, the total population of Tagong Township in Kangding County was 8837 , with a poor population of 1702 , and the incidence of poverty was $19.26 \%$. In the township, among those 1702 poor people, 425 people were trapped into poverty not long ago, wheres the remaining 1277 people were kept in poverty for a long period, indicating the chronic poverty incidence rate was $75 \%$. Tagong Township was more than 140 kilometers away from Kangding County, and the incidence of poverty was $19.26 \%$, far higher than the average level of Kangding County. In addition, through the survey we found that along the Tumen tour line, some residents had been out of poverty. However, in the areas away from the tourist attraction, poverty state was much worse.

In fact, not only in the two ethnic counties cited in the paper, in many other ethnic rural areas, chronic poverty problems also exist. Chronic poverty in ethnic rural areas lasts long, and the degree of poverty shows the increasing trend of transmission from urban center to village. the farther away from the urban and market areas, the longer the duration of poverty, the more serious the problem of chronic poverty.

4.3.2 Inter-generational Transmission of Poverty Was Prevailing Poverty theory of intergenerationalism suggests that poverty can be passed on to the next generation by the parental generation so that the former repeats the poverty status as the latter after adulthood, that is, the inheritance and reproduction of poverty in the intergenerational sense. The phenomenon of intergenerational transmission is a typical feature of chronic poverty, which is obviously higher than that of non-ethnic rural areas. In Sansha Village of Tagong Township, Kangding County, we visited 10 herdsman households and found that a majority of households were in poverty for many generations, thereafter intergenerational transmission of poverty was very obvious. This is mainly manifested in the following aspects. Descendants inherited yak, tents, horses and other economic and physical assets from their predecessors, and perpetuated parental production methods and lifestyles, continuing to make a living by traditional grazing. There were at least two children and one old man in each family. The belief that the more children you have, the more blessed you will be has been deeply rooted in local people's hearts, and local residents thought the more children they had, the more respectful they would be. However, the truth is that the more children you have, the more money and resources you need, and therefore the more difficult it is to satisfy the requirements for nutrition and medical care of children, resulting in the consequence that education and care for children can not be well guaranteed. Due to the lack of good sanitation and disease prevention measures, hereditary diseases can not be effectively avoided, resulting in intergenerational transmission of human capital. The younger generation inherited grassland, mountains and other natural capital from their parents, unable to renew the production methods and techniques. Tibetan Buddhism and the 'more children, more blessings' culture were transmitted from generation to generation, making it less likely to younger generations to accept new ideas, new culture and values. Political capitals like social prestige and aspiration were passed on steadily, leading to a consequence that the non-poor occupied much poverty alleviation resource, whereas the poor were less likely to obtain development opportunities. 
4.3.3 The Fragility of Poverty Was Evident The chronic poor were greatly heterogeneous. They were either old people, children and widows who were vulnerable to deprivation due to a particular stage in the life cycle, or people who were discriminated against because of low social status, or people who were discriminated within the family, or people who suffered from health problems, physical and mental deficiencies, or people living in remote rural areas. When disasters and changes came, the ability of heterogeneous groups to resist the disasters and changes was very fragile. In Sansha Village of Tagong Township, Kangding County, by interviewing 10 herders, we found that farmers here were poorly educated, and many family members within households had varying degrees of disability, with limited income source, and greatly relying on digging grass, selling yak milk and carving scriptures as a source of household income. Moreover, eight herdsman households investigated were caught in long-term poverty because of the family catastrophe. There were five households deep into poverty because lots of money was spent on medical expenses for the elderly at home and even had to borrow large mounts of money. Three families lost income source because their breeding yaks died of disease. At the same time, due to the climate change and population increase, local wild Cordyceps has been basically extinct in recent years, which to a certain extent weakened livings of people who relied on Dictyophora as the primary source of income. The vulnerability of livelihoods is one of the important reasons for the long-term poverty and the high rate of coming back to poverty in ethnic rural areas, especially in ethnic rural areas.

\section{Policy Recommendations for Poverty Alleviation in Ethnic Rural Areas from the Perspective of Chronic Poverty}

Chronic poverty is extremely common in ethnic rural areas, which requires much attention and effort in the process of ethnic poverty reduction. Currently it is a must to pinpoint the instinctive source of chronic poverty in ethnic rural areas, on which basis effective measures can be taken to alleviate poverty.

5.1 Accurately Identify the Targets of Poverty Alleviation and Change the Traditional Thinking of Poverty Alleviation The real essence of poverty alleviation is the ability to help the poor out of poverty. The government should change the mentality of poverty alleviation, and change the 'blood transfusion' poverty alleviation into 'hematopoietic' poverty alleviation. Government in ethnic rural areas should be in accordance with the principle of classification, and accurately identify the poor. The poor should be divided into two categories, disabled people without labor and health people, and put the former into the civil rights and subsistence allowances system management. In addition, poor people with labor should be classified as the poor, and be trained for simple business abilities.

\subsection{Attach Great Importance to Spatial Poverty Factors and Effectively Eliminates Regional} Barriers to Communication Due to geographical constraints, underdeveloped transportation and communications, etc, it is difficult for residents in ethnic rural areas to contact the outsider world, leading to the long-term poverty. Only by paying attention to the constraint factors of spatial poverty and removing communicating barriers through building more roads and water conservancy facilities, can poverty gradually be got rid of in ethnic rural areas.

\subsection{Actively Develop Education and Health Care and Invest in the Development of Human} Capital Education is a long-term plan to get rid of poverty in ethnic rural areas. Due to low development levels of history development, society, geography and basic education, the educational awareness of residents in ethnic rural areas is weak, which leads to the low level of education and to a certain extent results in the situation that people in ethnic rural areas can not be synchronized with modern culture. Therefore, in order to get rid of poverty, all levels of education should be actively developed. First of all, basic and vocational education should be intensively developed, enabling children to receive better education. Second, adult education should also be vigorously developed in ethnic rural areas, enabling adults to have a better access to the outsider world. In addition, health and other basic public services should be improved to effectively prevent the spread of infectious diseases. 5.4 The Government Should, on the Basis of Local Conditions, Vigorously Introduce Various Projects Beneficial for Residents Rely solely on government support is far from enough 
to alleviate poverty in ethnic rural areas. Foreign investment should be actively introduced in ethnic area to develop local economy, by which means local people's employment problems can be better solved. Only when the economy develops, will more opportunities and stronger ability to engage in social construction enrich income sources of the poor, on which basis poverty is likely to be eliminated.

\section{References}

[1] S. Andrew, Tackling Chronic Poverty, Population and Development Review, Vol. 5, pp. 136-138, 1997.

[2] J. Jalan, M. Ravallion, Determinante of transient and chronic poverty: Evidence from rural China, Word bank policy research working paper, 1998.

[3] Y. Zhang, G. H. Wan and Q. H. Shi, Whether temporary poverty in rural China is more serious, Global Economy, Vol. 1, pp. 144-160, 2012.

[4] J. S. Chen, Dynamics on chronic poverty research, Dynamics of Economics, Vol. 1, pp. 94-99, 2006.

[5] H. X. Lan, Study on the problems of chronic poverty in ethnic rural areas--Based on empirical analysis of Liangshan Yi Autonomy Prefecture, Soft Science, Vol. 6, pp. 73-78, 2013.

[6] L. Wang, Y. M. Xie and F. Z. Zhou, Research on Poverty Reduction under Multi - capital Constraints in Ethnic Poverty - stricken Areas, Qinghai Ethnic Study, Vol. 3, pp. 66-69, 2013.

[7] National Bureau of Statistics Household Survey Office, Poverty Monitoring Report of rural China in 2011, Beijing: China Statistics Press, 2010. 